\title{
Haemorrhagic shock encephalopathy syndrome presenting with myoglobinuria
}

\author{
Norbert Teig, Thomas G Nuesslein
}

\begin{abstract}
An infant with haemorrhagic shock encephalopathy syndrome (HSES) who in addition presented with hyperpyrexia and myoglobinuria is reported. As rhabdomyolysis is a feature of heat stroke and malignant hyperthermia, the association of HSES with myoglobinuria supports the hypothesis that HSES may be a form of hypermetabolic state triggered by hyperthermia.

(Arch Dis Child 1996; 74: 168-169)
\end{abstract}

Keywords: haemorrhagic shock encephalopathy syndrome, rhabdomyolysis, dantrolene.

\section{Case report}

A previously healthy 7 month old girl was found in the evening by her parents unresponsive and with shallow breathing after they had put her to bed about two hours before. The girl was seen by our emergency team at home with irregular breathing and intermittent clonic jerks. She was tightly wrapped with a rectal temperature of $40 \cdot 8^{\circ} \mathrm{C}$. Intravenous fluid administration was started and the infant was transferred to our intensive care unit after nasotracheal intubation.

The rectal temperature was $38.6^{\circ} \mathrm{C}$ on admission 60 minutes after the rectal administration of $250 \mathrm{mg}$ paracetamol and external cooling during transport. The extremities were warm, capillary refill was prolonged, skin turgor was normal, and mucous membranes were moist. The infant had marked tachycardia $(170-190 / \mathrm{min})$ and a deep breathing pattern. There were no organomegaly, rash, or signs of antecedent trauma. Pulsoxymetric oxygen

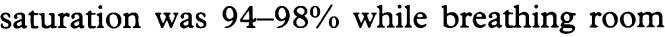
air. She had no spontaneous movements and

Children's Hospital of the Ruhr-University, St-Josef-Hospital, Alexandrinenstr 5, D-44791 Bochum, Germany N Teig

T G Nuesslein

Correspondence to: Dr Teig.

Accepted 13 September 1995

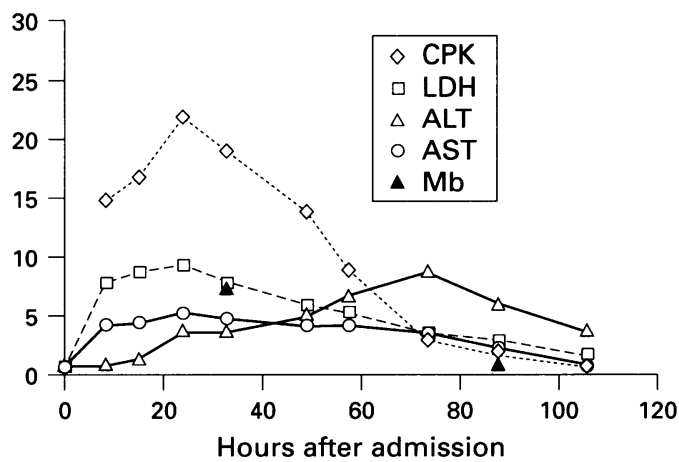

Serum values for creatine phosphokinase (CPK), lactate dehydrogenase $(L D H)$, alanine aminotransferase $(A L T)$, aspartate aminotransferase (AST), and myoglobin (Mb) in our patient. Values are given as multiples of the upper in our pati. responded weakly to pain stimuli, no verbal response, and no visual reaction could be evoked on neurological examination. Muscle tone was increased, especially in the arms, tendon reflexes were normal, and clonus could not be provoked. Meningeal signs were absent.

Abnormal results of blood chemistry are shown in the figure and table. The first urine specimen four hours after admission revealed no leucocytes or erythrocytes on microscopic examination but a strong reaction for haemoglobin and protein on dipstick analysis. Analysis of the red-brown discoloured urine showed markedly increased concentrations for myoglobin. Serum myoglobin on day 2 was raised to more than $700 \mathrm{ng} / \mathrm{ml}$ (upper limit $85 \mathrm{ng} / \mathrm{ml}$ ). Normal values were demonstrated for cerebrospinal fluid analysis (cell count, protein, glucose), C reactive protein, total protein, sodium, calcium, plasma carnitine, cholinesterase, ammonia, and urinary organic acids.

Doppler ultrasound one hour after admission showed normal flow patterns of the great intracerebral arteries; 24 hours later diastolic backflow in the anterior cerebral artery and slit ventricles were demonstrated. Electroencephalography tracings in the first two days showed a burst-suppression pattern. Cardiac ultrasound on admission demonstrated normal anatomy and function. No arrhythmias were noticed on continuous electrocardiographic monitoring. Bacterial cultures of blood, urine, and cerebrospinal fluid taken before the administration of antibiotics remained sterile.

\section{CLINICAL COURSE}

The girl passed bloody stools for two days, and the discoloration of urine was present from day 1 to day 3. Arterial blood pressure declined over the first 24 hours despite of aggressive volume replacement. The administration of high doses of catecholamines restored blood pressure and urine production towards normal. Rectal temperature fell parallel to

Blood chemistry of the patient studied

\begin{tabular}{|c|c|}
\hline \multicolumn{2}{|c|}{ Abnormal serum parameters on admission } \\
\hline \multicolumn{2}{|c|}{ pH } \\
\hline Carbon dioxide tension & $4.0 \mathrm{kPa}$ \\
\hline Creatinine & $115 \mu \mathrm{mol} / \mathrm{l}$ \\
\hline Potassium & $6.3 \mathrm{mmol} / 1$ \\
\hline \multicolumn{2}{|c|}{ Abnormal values during disease course } \\
\hline$\alpha_{1}$-Antitrypsin & $1 \cdot 2 \mathrm{~g} / 1$ (day 2$)$ \\
\hline Haptoglobin & $0.25 \mathrm{~g} / \mathrm{l}$ (day 3$)$ \\
\hline Uric acid & $0.66 \mu \mathrm{mol} / 1$ (day 3$)$ \\
\hline Antithrombin-III & $37 \%$ (day 2 ) \\
\hline Fibrinogen & $0.22 \mathrm{~g} / \mathrm{l}$ (day 2$)$ \\
\hline Prothrombin time & $9 \%$ (day 2 ) \\
\hline Partial thromboplastin time & 140 seconds (day 2$)$ \\
\hline Haemoglobin & $62 \mathrm{~g} / 1$ (day 3$)$ \\
\hline Platelet count & $5 \times 10^{9} / 1$ \\
\hline
\end{tabular}


blood pressure with hypothermic values recorded 12 hours after admission. Despite improving circulation our patient remained in a deep coma and without spontaneous breathing. The administration of mannitol $1 \mathrm{~g} / \mathrm{kg}$, in view of the evidence from Doppler ultrasound of increased intracranial pressure, did not alter her neurological condition. After five days the patient showed clinical evidence of cerebral death and a flat-lined electroencephalogram over 30 minutes. Forty eight hours later there was still evidence for cerebral death as assessed by two independent neurologists and we discontinued respiratory and inotropic support with parental consent. Necropsy was refused by the parents.

\section{Discussion}

Our patient exhibited the classical signs of haemorrhagic shock encephalopathy syndrome (HSES) as defined in the literature. ${ }^{12} \mathrm{We}$ diagnosed acute rhabdomyolysis by demonstrating myoglobin in the first urine specimen taken four hours after admission, and raised myoglobin in serum was still detectable on day 3. Creatine phosphokinase values rose to a maximum on day 2 and lactate dehydrogenase, hydroxybutyrate dehydrogenase, alanine aminotransferase, and aspartate aminotransferase were also raised (see figure).

To our knowledge this is the first report showing rhabdomyolysis with myoglobinuria in a patient with HSES. The clinical findings of HSES in concert with acute non-traumatic rhabdomyolysis and a hyperdynamic shock state in our patient are strikingly similar to those found in severe heat stroke, neuroleptic malignant syndrome, and malignant hyperthermia. It was proposed that hyperpyrexia, encephalopathy, and shock syndrome should be the proper name for this syndrome. ${ }^{3}$

We conclude that acute rhabdomyolysis does occur in HSES and we speculate that hyperthermia and/or hypermetabolism may be associated in some way with this event. The hyperpyrexia without any sign of infection in our patient may have been a result of external overheating, tight wrapping, or poor temperature regulation. Interestingly our patient had not had a raised temperature before and it is conceivable that she was faced with temperature stress for the first time. The metabolic decompensation that developed subsequently may have been due to overheating alone as in heat stroke, due to a concomitant metabolic defect as in malignant hyperthermia or due to a central dysregulation as in malignant neuroleptic syndrome. Previously a series of 10 patients was reported with only one patient surviving without major neurological sequelae, and this patient had been treated with dantrolene early in the course of his illness. ${ }^{2}$ Dantrolene blunts muscle cell hypermetabolism and is a proved and safe treatment for malignant hyperthermia. ${ }^{4}$ Case reports show evidence for the benefits of dantrolene in severe heat stroke $^{5}$ as well as in malignant neuroleptic crisis. ${ }^{6}$ In face of the high mortality and severe neurological sequelae in the remaining survivors, early use of dantrolene should be investigated as a possible treatment in patients suspected to suffer from HSES.

1 Bacon CJ, Hall SM. Haemorrhagic shock-encephalopathy syndrome in the British Isles. Arch Dis Child 1992; 67: 985-93.

2 Chaves-Carballo E, Montes JE, Nelson B, Chrenka BA. Hemorrhagic shock and encephalopathy. Clinical definition of a catastrophic syndrome in infants. Am $\mathcal{F}$ Dis Child 1990; 144: 1079-82.

3 Corrigan J. The ' $\mathrm{H}$ ' in hemorrhagic shock and encephalopathy syndrome should be 'hyperpyrexia'. Am $\mathcal{f}$ Dis Child 1990; 144: 1077 .

4 Kaus SJ, Rockoff MA. Malignant hyperthermia. Pediatr Clin North Am 1994; 41: 221-37.

5 Lydiatt JS, Hill GI. Treatment of heat stroke with dantrolene. FAMA 1981; 246: 41 .

6 Bismuth $\mathrm{C}$, Elkkaharat $\mathrm{D}$. Theoretical indications of dantrolene in neuroleptic malignant syndrome: efficacy in three cases. Vet Human Toxicol 1982; 24 (suppl): 52. 